\title{
Analizando el desarrollo de la creatividad en entornos educativos Una herramienta metodológica basada en el diseño.
}

\author{
Mª Belén Calavia Ferrández, Teresa Blanco Bascuas, Roberto Casas Nebra \\ Afiliación: HOWLab (Human OpenWare Research Lab) \\ Instituto de Investigación en Ingeniería de Aragón (I3A) \\ Universidad de Zaragoza, Mariano Esquillor s/n, 50018, Zaragoza, Spain. \\ Tel. +34-976762707, e-mail: 646000@unizar.es
}

\begin{abstract}
Resumen
Ante la necesidad detectada de aplicar la creatividad en los colegios, se aborda el diseño y desarrollo de un kit basado en el aprendizaje por retos, que busca fomentar los principales factores de la creatividad. Está formado por un conjunto de herramientas abiertas, accesibles e intuitivas basadas en el diseño, las cuales conectan los contenidos con los intereses y la realidad del alumno, generando nuevas posibilidades de aprendizaje competencial.
\end{abstract}

\section{¿Qué es la creatividad?}

¿Por qué las escuelas no producen "personas más creativas"? Esta fue una pregunta planteada por Guilford en 1950 (1). Pudiéndose afirmar, que dicha cuestión sigue sin tener respuesta actualmente.

La creatividad se define como un proceso esencialmente combinatorio. Es la capacidad de conectar los conocimientos aprendidos, para resolver problemas y crear cosas (2). Además, nos permite adelantarnos y buscar más allá de las pautas conocidas o los caminos establecidos (3). Es necesario resaltar que la capacidad creativa no es un talento limitado a algunas personas, sino que está presente en mayor o menor medida, en todos los seres humanos (1). Por tanto, la creatividad es un potencial que todos poseemos, y para despertarla, estimularla y desarrollarla requiere de la educación $\mathrm{y}$ de un entrenamiento adecuado en un ambiente propicio (4).

\section{Creatividad en educación}

Los entornos educativos pueden ser un buen entorno para estimular la creatividad, ya que es un lugar donde se imparte educación y enseñanza, las personas pasan gran parte de su tiempo y se puede desarrollar para todo tipo de clases sociales y en masas de estudiantes (5). En esta línea, es necesario resaltar la importancia de aplicar la creatividad de manera transversal dentro del currículo educativo (6).

\section{Aportaciones desde el diseño y la tecnología}

A partir de la investigación realizada se ha detectado la necesidad de desarrollar productos y servicios que favorezcan la creatividad. Las áreas de ingeniería de diseño y de tecnología implican un importante punto de partida en el desarrollo, ya que suponen una base de conocimiento y de recursos que pueden ser extrapolados y adaptados al ámbito educativo (Ver Figura 1).

Por un lado, el diseño puede mejorar la creatividad en el ámbito de la educación ya que proporciona a los estudiantes una mente más abierta, y crea un contexto que fomenta la creatividad como un elemento transversal, utilizando el conocimiento de todas las materias para resolver problemas (7).

Por otro lado, las nuevas tecnologías han alterado la enseñanza y el aprendizaje, con innovaciones y posibilidades para crear y compartir ideas y contenido. Se debe considerar el desarrollo y el impacto de la tecnología en el aprendizaje junto con las oportunidades de una educación creativa (8). Además, el mundo en el que vivimos de objetos interconectados, en el Internet of Things (9) supone un marco para desarrollar productos y servicios que se introduzcan en las aulas.

\section{Herramienta "Aprendizaje por retos"}

Se lleva a cabo una investigación de dos líneas teórica y práctica, mediante la identificación de problemas, oportunidades y nichos de cómo la creatividad está siendo aprendida, entendida y aplicada por los profesores en la actualidad. En este sentido, combinado con metodologías etnográficas y de observación, se comparan varios materiales educativos de aula, concluyendo que hay algunos factores de creatividad que no se están considerando (Ver Figura 2). Teniendo en cuenta esta realidad, se desarrolla un nuevo kit destinado a mejorar los principales factores de creatividad (Ver Figura 3).

Revista "Jornada de Jóvenes Investigadores del I3A", vol. 6 (Actas de la VII Jornada de Jóvenes Investigadores del I3A - 8 de junio de 2018). ISSN 2341-4790. 
Incluye las herramientas necesarias para realizar un estilo de "enseñanza por retos", tratando de resolver los desafíos de manera creativa; apoyándose en herramientas abiertas, accesibles e intuitivas basadas en el diseño; conectando los contenidos con los intereses y la realidad del alumno; y generando nuevas posibilidades de aprendizaje competencial. El kit ha sido evaluado de forma cualitativa $\mathrm{y}$ cuantitativa, para evidenciar su eficacia y para obtener retroalimentación necesaria para la mejora.

\section{Conclusiones}

Ante la necesidad detectada de aplicar la creatividad en los colegios, este proyecto presenta un estudio (teórico y práctico) que ha permitido recopilar conocimiento y detectar múltiples oportunidades y nichos. Posteriormente, se ha realizado un proceso de definición e ideación, siendo el resultado un kit de "Enseñanza por retos".

Con la finalidad de hacer tangible el resultado final, se ha creado un prototipo para poder evaluarlo y obtener retroalimentación para mejorarlo. A partir de la evaluación se puede aseverar que la herramienta comparte las mismas líneas y sigue los principios del currículo. Además, se reconoce de gran ayuda para fomentar la creatividad en los colegios, ayudando a los maestros a "producir personas más creativas".

\section{REFERENCIAS}

1. GUILFORD, Joy Paul. Creativity. American Psychologist, 1950, vol. 5, no. 9. pp. 444-454.

2. KLEIMAN, Paul. Towards Transformation: Conceptions of Creativity in Higher Education. Innovations in Education and Teaching International, 2008, vol. 45, no. 3. pp. 209-217.

3. DE BONO, Edward. El Pensamiento Creativo., 1994.

4. LÓPEZ MARTÍNEZ, Olivia. Enseñar Creatividad: El Espacio Educativo. Cuadernos De La Facultad De

Humanidades Y Ciencias Sociales.Universidad Nacional De Jujuy, 2008, no. 35. pp. 61-75.

5. SHAHEEN, Robina. Creativity and Education. Online Submission, 2010, vol. 1, no. 3. pp. 166-169.

6. FASKO, Daniel. Education and Creativity. Creativity Research Journal, 2001, vol. 13, no. 3-4. pp. 317-327.

7. THORSTEINSSON, Gisli; and PAGE, Tom. Teaching Creativity Across the Curriculum through Design Education. Case Studies. Educatia 21, 2017, no. 15. pp. 13-22.

8. HENRIKSEN, Danah; MISHRA, Punyaand FISSER, Petra. Infusing Creativity and Technology in 21 st Century Education: A Systemic View for Change. Educational Technology \& Society, 2016, vol. 19, no. 3. pp. 27-37.

9. PERERA, Charith, et al. Context Aware Computing for the Internet of Things: A Survey. IEEE Communications Surveys \& Tutorials, 2014, vol. 16, no. 1. pp. 414-454.

\section{FIGURAS}

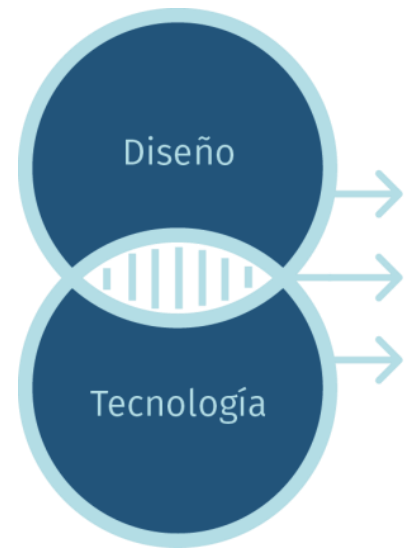

Formar personas más creativas

Figura 1. Objeto del proyecto

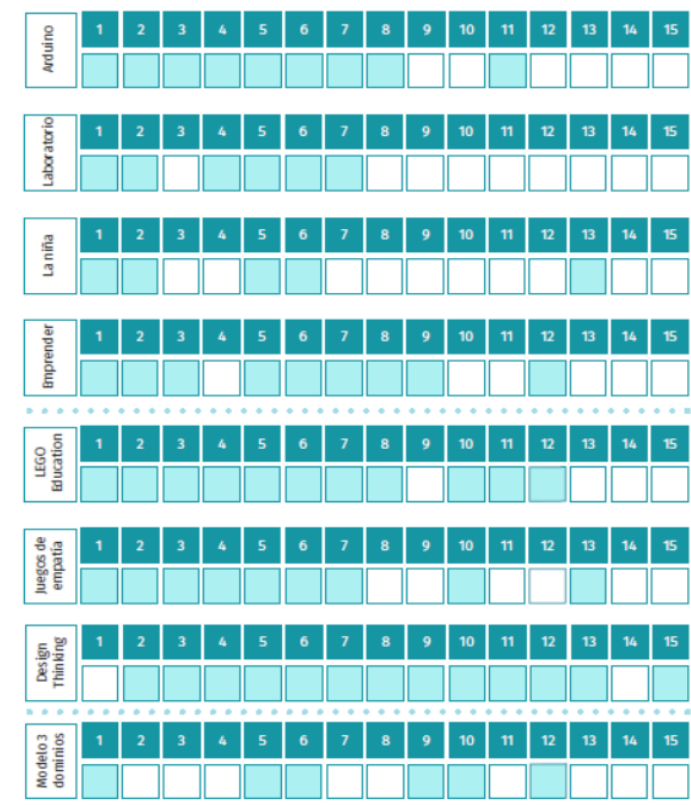

Figura 2. Factores de la creatividad de los materiales analizados

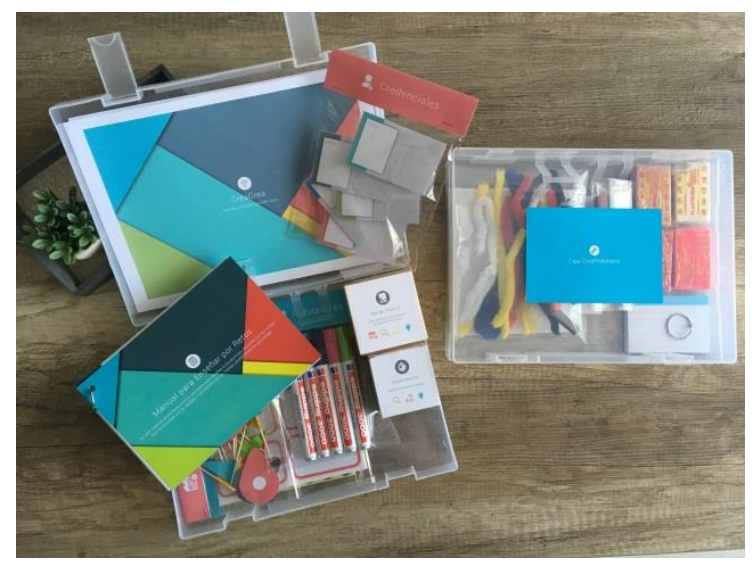

Figura 3. Prototipo Kit de creatividad

Revista "Jornada de Jóvenes Investigadores del I3A", vol. 6 (Actas de la VII Jornada de Jóvenes Investigadores del I3A - 8 de junio de 2018). ISSN 2341-4790. 